\title{
La helicicultura como alternativa de desarrollo sostenible: un estudio de caso ${ }^{*}$
}

\author{
Heliciculture as a Sustainable Development Alternative: A Case Study \\ Ricardo Alonso Colmenares-Florez iD \\ Doctorando en Administración Gerencial, Corporación Universitaria U de Colombia, \\ Medellín-Colombia, docentecolmenares@gmail.com \\ María Esther Alcántara-Gutierrez iD \\ Doctora en Dirección y Mercadotecnia, Universidad Benito Juárez, \\ Puebla-México, mariaestheralcantara@gmail.com
}

\begin{abstract}
Cómo citar / How to cite
Colmenares-Florez, R. A.; Alcántara-Gutierrez, M. E. (2021). La helicicultura como alternativa de desarrollo sostenible: un estudio de caso. Revista CEA, v. 7, n. 14, e1590. https://doi.org/10.22430/24223182.1590
\end{abstract}

Recibido: 30 de enero de 2021

Aceptado: 30 de abril de 2021

\section{Resumen}

El presente artículo tiene por objetivo determinar los beneficios que ofrece la helicicultura en el ámbito internacional. Para ello, se presenta un estudio de caso que fue aplicado en el municipio de San Vicente Ferrer (Antioquia), siendo este un referente en el manejo que se le ha dado a la helicicultura como actividad económica. Para ello, a través de la aplicación de una metodología cualitativa, esta investigación empleó un procedimiento exploratorio y descriptivo, el cual plantea la comparación de los efectos obtenidos que aún no han sido descubiertos para el bienestar de la sociedad. El estudio de caso, partiendo desde la revisión documental en función de las variables estéticas, biológicas, económicas, sociales y ambientales, arrojó como resultado que los beneficios de la helicicultura transcienden más allá de la variable económica, siempre y cuando se adopte un modelo eficiente de producción. Finalmente, luego del análisis realizado, se concluyó que la actividad es poco conocida en el medio, que se requieren permisos y licencias sanitarias de buenas prácticas para la cría del molusco Helix aspersa máxima, pero sobre todo de un conocimiento, tanto del modelo de producción sostenible en función de lo que representa la inversión, la infraestructura, la capacitación, el estudio de factibilidad financiera y las acciones de desarrollo, así como del estatus global del mercado, esto con la intención de consolidar esfuerzos para potencializar el comercio exterior, aprovechando la demanda de este producto en el mercado europeo y anglosajón.

Palabras clave: helicicultura, agroindustria, Helix aspersa, producción sostenible.

\footnotetext{
* Este artículo se deriva del proyecto de investigación de la tesis doctoral titulada «La helicicultura como alternativa de desarrollo sostenible. Caso: Municipio San Vicente de Ferrer» y ha sido financiado con recursos propios.
} 
Clasificación JEL: D10, D13, G10.

\title{
Highlights
}

- La helicicultura se convierte en la ganadería futurista, puesto que no afecta la fertilidad de los suelos.

- La helicicultura da una solución no solo económica, sino que también ofrece beneficios bajo el modelo de desarrollo sostenible.

- La principal estrategia de la helicicultura se encuentra en la adopción del modelo innovador de pronto engorde.

\begin{abstract}
This case study aims to determine the benefits that heliciculture can offer at the international level. It was conducted in San Vicente Ferrer (Antioquia, Colombia), which is a model in the use of heliciculture as an economic activity. Applying a qualitative methodology, this exploratory and descriptive study uncovers positive effects of heliciculture that could contribute to the well-being of society. The first stage in this study was a literature review to determine the aesthetic, biological, economic, social, and environmental variables of heliciculture, which has benefits beyond economic considerations as long as an efficient production model is adopted. Finally, after the analysis, it was concluded that heliciculture is little known in Colombian agriculture, and sanitary permits and licenses for good practices to farm Helix aspersa maxima should be created in the country. Above all, there is a knowledge gap in sustainable snail farming models in terms of investment, infrastructure, training, financial feasibility studies, and development actions, as well as the global status of this market. Therefore, efforts should be made to boost the foreign trade of this product, exploiting the demand for it in European and English-speaking markets.
\end{abstract}

Keywords: Heliciculture, agroindustry, Helix aspersa, sustainable production.

JEL classification: D10, D13, G10.

\section{Highlights}

- Heliciculture has become cutting-edge stockbreeding because it does not affect soil fertility.

- Not only economical, heliciculture also offers benefits in a sustainable development model.

- The main strategy of heliciculture is an innovative model called Fast Growth.

\section{INTRODUCCIÓN}

Según la Organización de las Naciones Unidas para la Alimentación y la Agricultura (FAO, 2016), la alimentación y la agricultura deben ser el objetivo principal de los planes de gobierno en cada uno de los estados en el mundo, sin dejar de lado las consecuencias ocasionadas por el cambio climático, donde se estima que para el año 2030, la población que convive en la pobreza ascendería de 35 a 122 millones de habitantes. Por tal motivo, pensando en su bienestar (Meiselman, 2016), las sociedades en el mundo requieren de alternativas para mejorar su calidad de vida en diferentes ámbitos. 
En el caso de Colombia, país que ha ido superando el conflicto armado, como principal objetivo de la firma definitiva del acuerdo de paz con las Fuerzas Armadas Revolucionarias de Colombia (FARC-EP) (Cairo et al., 2018), se proyectan oportunidades que generen alternativas sostenibles que contribuyan a resolver la problemática social, económica y ambiental (Criollo-Uyaguari et al., 2020), tal como lo plantea la teoría del modelo de desarrollo sostenible expuesta por De Vincentiis. Para Martínez (2016), el mercado de la helicicultura en el mundo experimenta un consumo anual de 300000 toneladas de caracoles terrestres, pero donde la producción solo alcanza para 35000 toneladas, generando un déficit de 56000 toneladas pendientes por abastecer a un mercado por explorar.

Según Gutiérrez y Guzmán (2009), la helicicultura es una actividad económica del sector agropecuario que puede diversificar la productividad en zonas rurales como el municipio de San Vicente Ferrer como una solución a la problemática del territorio afectado por la violencia. El presente artículo busca determinar, desde el modelo de desarrollo sostenible, cada uno de los efectos de la helicicultura, tanto en la población desplazada, como en el grupo de desmovilizados en San Vicente de Ferrer (Gallopín, 2003).

Al revisar diversas fuentes documentales, se evidenció un trabajo desarrollado por López-Palechor et al., (2017), donde determinan que la helicicultura es una actividad rezagada por los problemas del conflicto armado. Lo anterior, llevó al presente trabajo a dar una mirada diferente de cara a los acuerdos del proceso de paz en Colombia, siendo esta una alternativa sostenible para la población tanto víctima como victimaria durante la confrontación.

El objetivo general de este trabajo es determinar las bondades de la helicicultura a nivel internacional, tomando como punto de partida conocer el funcionamiento helicícola y la dinámica del mercado en función de la oferta y la demanda del caracol Helix aspersa máxima, chequeando la oportunidad y la viabilidad de la actividad económica. Pero no solo basta conocer el mercado: lo atractivo de la helicicultura es el modelo innovador de productividad en cada uno de los lotes, optimizando de esta manera los recursos requeridos en dicha actividad económica.

\section{MARCO TEÓRICO}

\section{Modelo del desarrollo sostenible}

Los antecedentes detallan tanto los estudios como los modelos de gestión vinculados al problema planteado, con perspectiva de desarrollo local y regional.

Según De Vincentiis (2012) y Abay y Jensen (2020), para una explotación sostenible, el consumo racional y apropiado de los recursos naturales depende de la producción pecuaria, sobre todo, del control de contaminantes para su debido funcionamiento. Estos son temas que deben ser tratados por cada gobierno. Es ahí donde se contempla la oportunidad de diseñar una propuesta alternativa de la actividad helicícola, bajo un modelo emprendedor y eficiente para víctimas y victimarios que fueron beneficiados con el proceso de paz en el departamento de Antioquia, ya que serán reincorporadas a la vida civil, lo cual requiere de un sinnúmero de opciones para pensar en una paz duradera y convincente. No solo considerar una alternativa que genera beneficio económico y social, 
sino pensar en un escenario que vincule de manera integral esta acción de gestión como alternativa en otras partes del mundo.

Según Artaraz (2002) y Padilla (2002), con la teoría del desarrollo sostenible se busca mejorar un modelo de helicicultura que garantice la sostenibilidad de los recursos naturales empleados, así como el mejoramiento del desarrollo social y económico de una comunidad. Pero no se puede pensar en desarrollo sostenible sin visionar un modelo agropecuario que contribuya a favorecer los recursos naturales de un territorio.

\section{Modelo agropecuario para el desarrollo}

El informe sobre el desarrollo mundial (Fantom et al., 2016), fundamenta al sector rural, ya que el $78 \%$ de la población pobre del mundo depende de la agricultura como medio de subsistencia. Según el Fantom et al., (2016), aumentar la productividad y resiliencia de los establecimientos agropecuarios es el principal objetivo de las propuestas gubernamentales en una región, además de fortalecer los vínculos entre los campesinos y los mercados, ofreciendo productos a un precio accesible con el propósito de impulsar la prosperidad.

El programa de ganadería del Banco Mundial se centra en una ganadería futurista en relación con los factores abióticos, la bondad nutricional, fortalecimiento de las cadenas de valor y sobretodo la creación de empleo. Según la FAO (2016), el sector rural colombiano es uno de los que más potencialidad tiene en América Latina, ya que cuenta con una mayor capacidad de ser despensa pecuaria en el mundo.

De acuerdo con las estadísticas actuales, de los 45.4 millones de hectáreas que están siendo utilizadas en Colombia, aproximadamente el $66 \%$ es de uso no adecuado, puesto que el $37 \%$ es sobre utilizado y el $29 \%$ subutilizado. Teniendo presente los estudios anteriores, y los realizados por la Universidad Nacional de Colombia, lo que esperan los victimarios reincorporados en el proceso de paz es poder participar económicamente en el sector de la ganadería.

La producción internacional de la helicicultura se encuentra resumida en la Tabla 1 , donde se abastece el consumo a nivel mundial.

Tabla 1. Competencia Internacional

Table 1. International competence

\begin{tabular}{ccc}
\hline País productor & Producción anual & Exportación \\
\hline Argentina & 30 toneladas & Del 70 a $75 \%$ de la producción \\
\hline Perú & 25 toneladas & $70 \%$ de la producción \\
\hline Ecuador & 25 a 30 toneladas & $40 \%$ de la producción \\
\hline
\end{tabular}

Fuente: elaboración propia a partir de (Martínez, 2016).

Según la FAO (2016), es importante repensar el modelo agropecuario que deben adoptar cada una de las regiones en el mundo. Con base en la tabla anterior, se evidencia la oportunidad en desarrollar un modelo de ganadería futurista que provea la carencia de la demanda actual en el mundo. Una vez concebido el modelo agropecuario, no se deben dejar de lado los factores necesarios que contribuyan al éxito de la productividad de un país. 


\section{Modelo del Diamante de Porter}

Según Kramer y Porter (2006) y Erboz (2020), a largo plazo la productividad nacional será el principal determinante del estándar de vida de un país. De acuerdo con esto, según Porter, no se puede desconocer lo que se observa en la Figura 1.

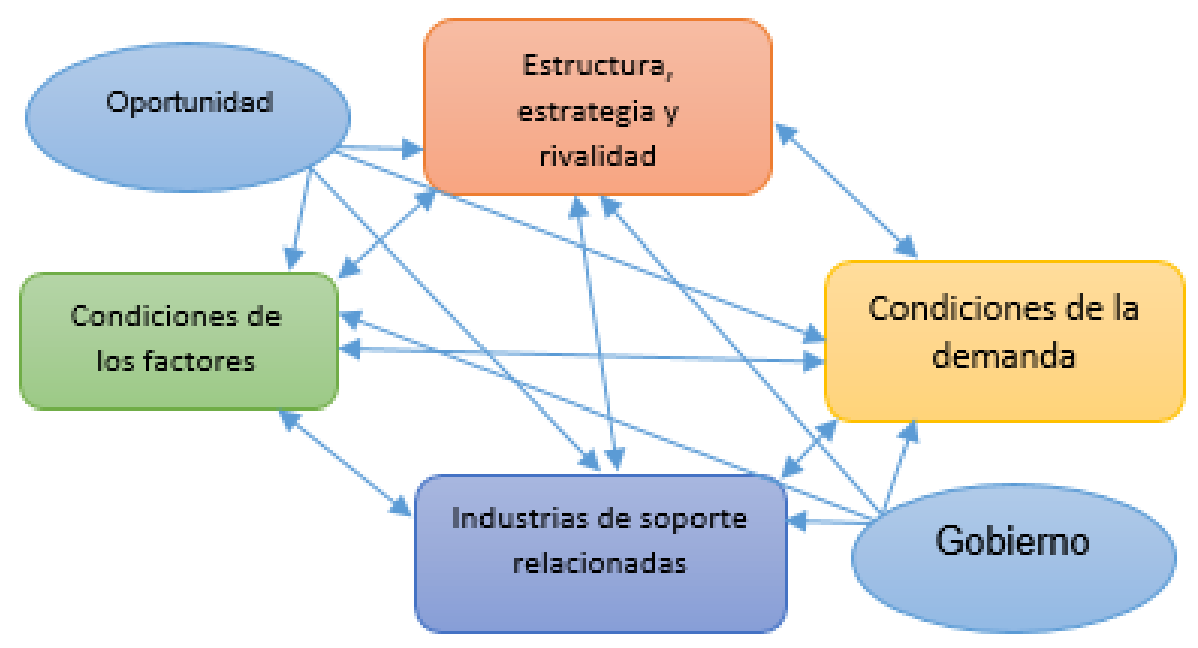

Figura 1. Modelo del Diamante de Porter

Figure 1. Porter's Diamond Model

Fuente: elaboración propia a partir de (Kramer \& Porter, 2016).
a. Condiciones de los factores.
b. Condiciones de la demanda.
c. Industrias de soporte relacionadas.
d. Estrategia, estructura y rivalidad de la empresa.

Según Kramer y Porter (2006), las dimensiones descritas en la anterior figura visionan una organización que pretenda incrementar el valor dado al cliente final, así como de optimizar los costos necesarios para la operación requerida en la actividad helicícola. Teniendo en cuenta el modelo teórico propuesto por Kramer y Porter (2016), la helicicultura para potencializar su productividad en el municipio de San Vicente Ferrer, en particular, debe tener presente los factores internos y externos que condicionan dicha actividad económica, el conocimiento del mercado potencial y de sus competidores en el ejercicio activo, así como la variable económica.

No se puede hablar de helicicultura sin tener claro el concepto de innovación para una actividad poco conocida en el medio. Según López-Palechor et al., (2017), se debe potencializar los anteriores componentes descritos en el modelo del Diamante en función de modernizar la helicicultura para que no sea concebida como una actividad rezagada en el sector primario de la economía colombiana.

\section{Innovación y emprendimiento}

Según Drucker (2004), el empresario innovador ve el cambio como una norma saludable, pero no necesariamente es él mismo el que lleva a cabo el cambio. A su vez busca el cambio respondiendo a 
él y lo visiona como una oportunidad, aumentando por ejemplo la productividad u optimización de los recursos por medio de potencializar un producto, entrenando el talento humano, diseñando modelos de producción, entre otros recursos más. No necesariamente se debe concebir la innovación como la invención de algo no existente ante los ojos del hombre, sino el mejoramiento o adecuación de lo existente optimizando su funcionalidad y servicio ante el mismo ser humano y su entorno. La helicicultura no es una actividad nueva: su explotación data de países como Marruecos desde los años 90. Lo que se pretende es modernizar el ejercicio en el municipio de San Vicente Ferrer para lograr determinar las diferentes bondades que tiene la helicicultura para una región.

Además, se deben tener presente elementos que contribuyan a la obtención de un resultado exitoso, de una búsqueda consciente y decidida de oportunidades, que son encontradas en pocas ocasiones. En este orden de ideas, según Drucker (2004), existen cuatro áreas de oportunidad dentro de una actividad:

- Acontecimientos inesperados: los éxitos y fracasos son fuentes fructíferas de oportunidades de innovación.

- Incongruencias: un desfase entre las expectativas y los resultados puede abrir opciones de innovar.

- Necesidades del proceso: revisión si existe carencia de acciones efectivas para el proceso a realizar.

- Cambios sectoriales y de mercado: estudiar y explorar nuevas opciones de mercado donde emerger.

\section{METODOLOGÍA}

Según Esterberg (2002), el método de investigación de tipo cualitativa se fundamenta desde la revisión literaria de diferentes fuentes documentales, como tesis doctorales sobre la producción de carne de caracol y su respectivo estudio de mercado, como de artículos de revista que evidencian los pros y contra de la helicicultura en las diferentes regiones al interior y exterior del territorio colombiano. Dentro de los autores, se logra evidenciar el desarrollo de la actividad helicícola en el territorio marroquí, así como en el continente europeo, con grandes resultados durante su productividad.

Según Valles (2000), la revisión de la literatura supone estar al día con lo publicado sobre el tema objeto de estudio, pero sobre todo con la utilización de las estadísticas existentes. Por otra parte, los autores determinaron que esta fase de la investigación no desconoce el importante manejo de estudios que generen un impacto social y ambiental. Según Arias (1999) y Caballero (2000), además de la revisión literaria sobre la actividad helicícola, la siguiente fase realizada es la observación en dos campos de producción del caracol Helix aspersa máxima en el municipio de San Vicente Ferrer, evidenciando el modelo de cultivo y producción actual. El encuentro de las revisiones, como de las observaciones en las anteriores fases de la investigación, brinda una oportunidad clara en el marco legal y arancelario para describir el protocolo de ampliar el mercado internacional. Las anteriores acciones contribuyeron al desarrollo y cálculo del estudio de viabilidad desde el manejo del costo unitario de producción, como de los resultados positivos (utilidades) en la ejecución del modelo propuesto para emprender con asertividad. 
La investigación finalizó con el análisis de las bondades y potencialidades que distinguen a la actividad de la helicicultura en una región. Teniendo en cuenta que el posconflicto es una oportunidad para una sociedad, a partir de las variables e indicadores que determinan las bondades en una situación y el desarrollo sostenible como modelo integral entre lo social, medioambiental y económico, relacionadas a continuación en la Tabla 2.

Tabla 2. Variables e Indicadores

Table 2. Variables and indicators

\begin{tabular}{ccc}
\hline Variable & Dimensión & Indicador \\
\hline \multirow{2}{*}{ Efecto de la helicicultura } & Estético & Cosmético \\
\cline { 2 - 3 } & Medio Ambiente & Eficiencia \\
\cline { 2 - 3 } & Económico Social & Manejo de Residuos \\
\cline { 2 - 3 } & Alimenticio & Emprendimiento \\
\cline { 2 - 2 } & & Información nutricional \\
\hline
\end{tabular}

Fuente: elaboración propia a partir de (FAO, 2016).

Las fuentes de investigación sobre la temática son insuficientes, partiendo de un estancamiento de la producción de caracol en Colombia, como lo plantean algunos autores previamente citados, se prevén alternativas que le garanticen a la población afectada un sustento sostenible, sobre todo, un impacto a la sociedad en general (Álvarez-Gayou, 2005).

\section{RESULTADOS}

En esta sección se presentan la revisión documental, análisis, modelación, resultados y recomendaciones acorde a los inconvenientes que ha tenido la helicicultura en Colombia. No solo basta con saber que es la helicicultura; el éxito de dicha actividad económica depende en ampliar aún más la comprensión del mercado nacional e internacional, repensando la oportunidad de exportar la producción. Producción que depende, en gran medida, del planteamiento de un modelo innovador que optimice los recursos necesarios para llevar a cabo dicha actividad, así como la descripción de los efectos sostenibles de la misma.

\section{Conocimiento del mercado}

De acuerdo con la revisión documental, la producción de la helicicultura está orientada a la obtención de huevos y caracoles. Si bien no es una actividad muy conocida en el mundo, en el sector primario de la economía de Colombia tiene cierta popularidad, razón por la que se propone como una alternativa viable y sostenible para fomentar el empleo de la población afectada por el conflicto armado en el departamento de Antioquia (Jiménez \& Rodríguez, 2007).

Según Lamprea Delgado et al., (2009), el desarrollo de la helicicultura debe estar orientada a crear una cultura de consumo nacional, hasta lograr niveles de producción que permitan acceder al mercado extranjero. Es importante tener presente que los principales consumidores son los países miembros de la Unión Europea (UE), tales como España, Francia, Grecia e Italia. Según Lamprea 
Delgado et al., (2009), el consumo anual de caracoles en el continente europeo asciende a las 150 mil toneladas, mientras que el consumo mundial en ese mismo tiempo es de 300 mil toneladas, lo que quiere decir que el potencial superior al $50 \%$ de la demanda total en el mundo se encuentra en los países europeos. Siendo optimistas, y de acuerdo con las proyecciones emitidas por el portal agropecuario argentino Agromeat, según Martínez (2016), se calcula que para los próximos veinte años el consumo mundial de caracoles terrestres alcance las 1500000 toneladas. Por otro lado, diversas investigaciones de mercado encuentran a Sudamérica como el principal abastecedor de caracol terrestre. En los países importadores de caracol terrestre, se cuenta aproximadamente con la siguiente referencia, tal como lo señala la Tabla 3.

Tabla 3. Países Consumidores

Table 3. Snail-eating countries

\begin{tabular}{ccc}
\hline País comprador & Consumo anual & Importación anual \\
\hline Francia & 65 mil toneladas & 25 mil toneladas \\
\hline Italia & 12 mil toneladas & 5 mil toneladas \\
\hline España & 14 mil toneladas & 5 mil toneladas \\
\hline Fuente: elaboración propia a partir de (Martínez, 2016).
\end{tabular}

Con base en lo anterior y, según Martínez (2016), existen otros países consumidores de este producto a parte de Francia, Italia y España, como Estados Unidos, Japón (y otros países asiáticos), cuyos principales exportadores son Yugoslavia, Turquía y Marruecos.

Luego de analizar los cálculos de la oferta y la demanda de caracol comestible en el mundo, se encontró que falta por abastecer 56000 toneladas al año, lo que a su vez significa la oportunidad de exportar esa cantidad anualmente, y la tendencia de consumo va en aumento, sobre todo los países que han aumentado la intención de importar este producto. Además, las proyecciones de demanda visionan una cifra para los próximos 5 años de 1,2 millones de toneladas en el mundo, según Villamil Kiewu (2010).

Es a partir del anterior análisis que se vislumbra una estrategia de gestión comercial que contribuya a diversificar el mercado de los productos pecuarios, así como el desarrollo regional que se dedique a esta producción económica.

De acuerdo con los costos comparados en la actividad helicícola, la Tabla 4 relaciona los países productores y exportadores de carne de caracol, donde se observa.

Tabla 4. Comparativo valor $\mathrm{CIF} / \mathrm{Kg}$

Table 4. Comparison of $\mathrm{CIF} / \mathrm{Kg}$ charges

\begin{tabular}{cc}
\hline País & Valor (U\$ / Kg / CIF) \\
\hline Marruecos & 1,7 \\
\hline Portugal & 2 \\
\hline Francia & 2,3 \\
\hline Argentina & 4 \\
\hline Colombia $\left({ }^{*}\right)$ & 4,9 \\
\hline
\end{tabular}

Fuente: elaboración propia a partir de (Martínez, 2016).

${ }^{*}$ ) costo en Colombia con el sistema de producción actual. 
Según López-Palechor et al., (2017), la incipiente capacidad financiera de los helicicultores y su costo promedio del kilo de caracol tan alto, como se evidencia en la Tabla 4, es una limitante para emprender en esta actividad económica; pero, con el comparativo anterior, es apreciable que el manejo estratégico de los costos del proyecto se convierta en un factor principal para replantear la proyección financiera de la helicicultura, optimizando dichas erogaciones.

\section{Manejo para la exportación}

Con el análisis de oferta y demanda internacional y con la evaluación de alternativas de la revisión documental para exportar el caracol en el mercado ibérico, Colombia cuenta con acuerdos comerciales con la Unión Europea que les facilita el acceso a las preferencias arancelarias. Para el caso de la exportación del caracol se puede tomar como partida arancelaria 037600000, lo que genera una ventaja del $0 \%$ de arancel, siendo un incentivo para pensar en la exportación al mercado europeo del caracol Helix aspersa máxima.

Además, se debe contar con lo establecido en el capítulo XI del Decreto 3075 de 1997, el cual hace referencia a los requisitos mínimos necesarios para realizar el proceso de exportación, como es la «expedición del certificado de inspección sanitaria», cuyo documento depende de:

- Copia del Registro Sanitario INVIMA.

- Acta de inspección de la mercancía.

- Resultados de los análisis de laboratorio (cuando el país importador así lo requiera).

Los helicicultores que alguna vez realizaron una exportación, al mismo tiempo que los importadores del continente europeo, adquieren el producto envasado con salmuera, el cual le da mayor conservación al producto de carne de caracol.

\section{Modelo Innovador}

De acuerdo con la metodología planeada en este artículo, se evidencia que en Colombia no es atractiva la helicicultura, debido al modelo actual que desarrollan. Según la Red Andaluza de Experimentación Agraria (2008), de las tres clases o estrategias productivas en la actividad de helicicultura, la más interesante se muestra en la Figura 2.

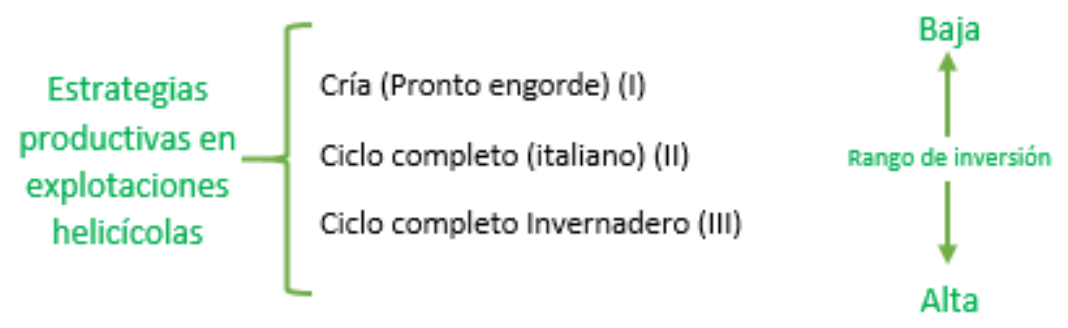

Figura 2. Clasificación de sistemas según estrategias vs inversión

Figure 2. Classification of snail farming techniques based on the size of the investment, from low to high Fuente: elaboración propia a partir de (Kramer \& Porter, 2016). 
En el caso colombiano, y teniendo en cuenta el bajo nivel de inversión, la elección sería por la estrategia de explotación (Cría (pronto engorde)), como se observa en la Figura 2. Por su parte, la Red Andaluza de Experimentación Agraria (RAEA) determina los factores esenciales para este tipo de estrategia de la siguiente manera:

a. Estructura del campo y su composición, (zonas de refugio y alimentación).

b. Suplementación estratégica.

c. Densidad de cría.

La evaluación de la inversión se calcula a través del umbral de rentabilidad, excedente de producción y la tasa interna de retorno de las estrategias (TIR).

Tabla 5. Comparación análisis de la inversión

Table 5. Analysis of the investment of three snail farming strategies

\begin{tabular}{cccc}
\hline Estrategia & Umbral $(\mathrm{kg})$ & Nivel de pcc $(\mathrm{kg})$ & TIR (\%) \\
\hline Pronto engorde & 5372 & 24730 & 12.3 \\
\hline Italiano & 5703 & 6156 & -5.3 \\
\hline $\begin{array}{c}\text { Intensivo en } \\
\text { Invernadero }\end{array}$ & 13347 & 20187 & 6.4 \\
\hline \multicolumn{2}{c}{ Fuente: elaboración propia a partir de (ABC del Finkero, 2013). }
\end{tabular}

Como se percibe en la Tabla 5, la estrategia de pronto engorde resumido por la RAEA, presenta una combinación apropiada donde se minimizan los riesgos, mientras que los beneficios económicos y técnicos se maximizan. De acuerdo con la propuesta de RAEA - Ganadería, las medidas necesarias para adoptar en la actividad de la helicicultura en el municipio de San Vicente Ferrer son:

a. Cuando los caracoles Helix aspersa máxima hayan completado su apariencia de ser huevo, deben permanecer en el campo hasta que se comercialice. (Su crecimiento, hasta su venta, debe permanecer en el mismo lugar).

b. Mantener una tasa de mortalidad de los caracoles no superior al $20 \%$ y una densidad final de 1.8 $\mathrm{kg} / \mathrm{m} 2$ a $3 \mathrm{~kg} / \mathrm{m} 2$ (que sería el ideal en condiciones climáticas favorables).

c. La explotación del criadero no será inferior a $10000 \mathrm{~m} 2$, lo que quiere decir una hectárea de suelo como mínimo; la Red Andaluza de Experimentación Agraria propone tener una persona de tiempo completo, a lo que en realidad el modelo colombiano se propone tener tres personas, teniendo presente que el factor humano no incide en el costo del producto, pero incentiva la generación de empleo.

d. La superficie se debe delimitar en 28 campos de 6 metros x 46 metros, dejando pasillos longitudinales de 1 metro y un pasillo central de 4 metros. Lo que quiere decir que la superficie efectiva de explotación es de $7728 \mathrm{~m} 2$.

La estrategia propone unas condiciones climáticas semiáridas mediterráneas, lo que se traduce en:

- Temperatura entre el rango de $15^{\circ} \mathrm{C}-22^{\circ} \mathrm{C}$.

- Humedad en el criadero diurno entre el $75 \%$ - 85\%.

- Humedad en el criadero nocturno entre el 85\% - 90\%. 
En estas condiciones, tal como se proyectarían ciclos productivos de 6 meses por lote de cría, se propone tener como mínimo dos lotes productivos, evidenciados en la Tabla 6.

Tabla 6. Plan proyectado de cría pronto engorde por lote

Table 6. Schedule of a Fast Growth [Pronto Engorde] project of two batches

\begin{tabular}{|c|c|c|c|c|c|c|c|c|c|c|c|c|c|c|c|c|c|c|c|c|c|c|c|c|}
\hline & \multicolumn{12}{|c|}{ AÑO 1} & \multicolumn{12}{|c|}{ AÑO 2} \\
\hline & 1 & 2 & 3 & 4 & 5 & 6 & 7 & 8 & 9 & 10 & 11 & 12 & 1 & 2 & 3 & 4 & 5 & 6 & 7 & 8 & 9 & 10 & 11 & 12 \\
\hline \multicolumn{25}{|l|}{ Lote 1} \\
\hline \multicolumn{25}{|l|}{ Preparación terreno } \\
\hline \multicolumn{25}{|l|}{ Huevos } \\
\hline \multicolumn{25}{|l|}{ Cría } \\
\hline \multicolumn{25}{|l|}{ Comercialización } \\
\hline \multicolumn{25}{|l|}{ Lote 2} \\
\hline \multicolumn{25}{|l|}{ Preparación terreno } \\
\hline \multicolumn{25}{|l|}{ Huevos } \\
\hline \multicolumn{25}{|l|}{ Cría } \\
\hline Comercialización & & & & & & & & & & & & & & & & & & & & & & & & \\
\hline
\end{tabular}

Fuente: elaboración propia a partir de (Eslava et al., 2006).

De esta manera organizada como se deben manejar los cultivos helicícolas, se garantizaría de un terreno de una hectárea (10 000 metros cuadrados), la superficie útil sería de 7800 metros cuadrados, aproximadamente, teniendo presente que la superficie restante forma parte de las áreas para el manejo del empaque del producto, preparación del alimento, bodegaje, pasillos entres lotes y otras áreas necesarias para ello.

Con el plan estratégico anterior, se obtendría una productividad por lote promedio entre 6250 y 6 300 kilogramos. Acorde con el precio promedio en el mercado de $\$ 20.000$ por kilogramo de carne de caracol Helix aspersa máxima, el resultado financiero bruto se percibe en la Tabla 7.

Tabla 7. Resultado bruto por año

Table 7. Annual gross income

\begin{tabular}{cc}
\hline Concepto & Año 1 \\
\hline Ingresos por ventas & 502320000 \\
\hline Costos fijos & 70427645 \\
\hline Costos variables & 215156214 \\
\hline Inversiones modelo «pronto engorde» $\left(^{*}\right)$ & 26464600 \\
\hline Utilidad Bruta & 190271541 \\
\hline Margen bruto & $37.88 \%$ \\
\hline
\end{tabular}

Fuente: elaboración propia a partir de (Chacón et al., 2010).

Nota. $(*)$ las inversiones para el modelo o sistema «pronto engorde» se amortiza por un período de 3 años.

Quiere decir que, al aumentar la producción de la carne de caracol, se logra optimizar el costo fijo, que de acuerdo con la Gerencia Estratégica de Costos (GEC), proyectada en la Tabla 7, conseguiría hacer atractivo el modelo para implementar la helicicultura en el sector rural, disminuyendo el valor $\mathrm{CIF} / \mathrm{Kg}$ en dólares, frente al valor obtenido bajo el modelo tradicional. De esta manera, Colombia lograría obtener un kilo de carne de caracol a 3.6 dólares y no a los 4.9 dólares en que se encuentra 
actualmente, esto con el modelo de pronto engorde debidamente adoptado en los cultivos de helicicultura (Bojö, et al., 1990).

De esta manera se logra maximizar la producción, disminuyendo el costo CIF frente a otros países productores de la carne de caracol Helix aspera máxima. El ideal está en salvaguardar un índice de mortalidad equivalente al $10 \%$ por lote.

\section{Efectos sostenibles de la Helicicultura}

Es importante tener presente las variables e indicadores determinadas por la helicicultura, que es objetivo de la investigación (Iglesias et al., 2012). De acuerdo con los diferentes efectos consultados que tiene la producción del caracol Helix aspera máximo, o Müller, desde otras aristas diferentes a la economía, se cuenta:

\section{Alimenticias}

Según Fleta Zaragonzano (2017), la antigua Roma consumían caracoles, no solo como alimento, sino que se consideraba un remedio eficaz para enfermedades estomacales y respiratorias, tal como lo manifestó «Plinio el Viejo» dentro de sus recomendaciones. Para entender mejor las ventajas del producto de la actividad helicícola, la Tabla 8 muestra un comparativo nutricional frente a la carne de res y pollo.

Tabla 8. Comparativo nutricional de la carne de caracol frente a la de res y pollo (100 grs) Table 8. Nutritional comparison of snail, beef, and chicken (100 grs)

\begin{tabular}{cccc}
\hline Nutriente & Caracol & Res & Pollo \\
\hline Calorías (kcal) & 75.0 & 168.0 & 85.0 \\
\hline Grasa (grs) & 0.8 & 10.0 & 3.0 \\
\hline Colesterol (mg) & 50.0 & 70.0 & 61.0 \\
\hline Sodio (mg) & 70.0 & 110.0 & 56.0 \\
\hline Carbohidratos (grs) & 2.0 & - & 2.0 \\
\hline Fibra (grs) & - & - & - \\
\hline Azúcares (grs) & - & - & - \\
\hline Proteínas (grs) & 15.0 & 19.0 & 14.3 \\
\hline Calcio (mg) & 170.0 & 11.0 & 7.0 \\
\hline Hierro (mg) & 35.0 & 3.0 & 0.7 \\
\hline Magnesio (mg) & 250.0 & 16.0 & 17.5 \\
\hline Vitamina A (mcg) & 30.0 & 20.0 & 12.0 \\
\hline Vitamina B12 (mcg) & 0.5 & 1.0 & - \\
\hline Vitamina C (mg) & - & - & 2.8 \\
\hline Fuente: elaboración propia a partir de (Fleta Zaragonzano, 2017).
\end{tabular}

Tal como lo expone Fleta Zaragonzano en su estudio, «la carne de caracol prácticamente aporta la totalidad de los aminoácidos necesarios para la alimentación de una persona», de acuerdo con la Tabla 8. Por otra parte, el caracol aporta menos cantidades de consumo de grasas en el cuerpo humano que la carne de res y de pollo, haciendo la carne de caracol más saludable para el consumo 
y sin ningún tipo de contraindicación. A raíz de su composición nutricional, muchos países como Portugal, Italia y España, entre otros, acostumbran a acompañar dentro de su dieta o alimentación saludable la carne de caracol con aceite de oliva, ajo y perejil para aprovechar al máximo sus beneficios nutricionales. Es ahí donde surge la oportunidad en crear una cultura de consumo interno en el territorio colombiano, para aumentar la demanda de este producto. Para ello se requiere de una campaña masiva por parte de la colectividad de helicicultores con un amplio conocimiento del tema, para darse a conocer al público a través de medios masivos publicitarios.

\section{Estéticas y terapéuticas}

Sin lugar a duda, son muchas las bondades nutricionales que ofrece la carne de caracol, pero son aún más los beneficios terapéuticos que la baba de caracol proporciona. Según Sastre (2006) y Fleta Zaragonzano (2017), la baba de caracol tiene propiedades curativas que sirven para sanar los tejidos. Además, la baba de caracol:

- Repara tejidos, no solo en la eliminación de manchas y quemaduras, sino que sus antioxidantes ayudan a reducir la aparición de líneas de expresión. Por otra parte, disminuye las marcas originadas por las estrías y cicatrices.

- Previene, por sus propiedades ayuda a combatir el acné, eliminando las bacterias de la piel, gracias a su efecto antibiótico.

- Ayuda a la tersura de la piel evitando su flacidez, gracias a su contenido de colágeno y elastina.

De acuerdo con los estudios publicados por la revista International Journal of Cosmetic Science, el caracol produce una secreción (SCA) basta en proteínas de alto y bajo peso molecular, ácido hialurónico y antioxidantes necesarios para las terapias regenerativas de los tejidos cutáneos dañados. Es de aclarar que todas las babas de caracol poseen estos beneficios. Los investigadores de fisiología y biología de la Universidad Autónoma de Madrid (UAM) tienen claro que la familia Helix aspersa cuenta con estos beneficios.

\section{Ambientales}

Según Artaraz, (2002), en el informe anual de los recursos biológicos, el caracol se puede alimentar de las especies nativas de flora como Wigginsia vorkeniana, producida por el medio ambiente. Pero es de aclarar que para obtener una producción para comercialización de manera óptima se optaría por la cerdo lactancia para su crecimiento en el menor tiempo posible.

Una de las principales bondades de este tipo de ganadería novedoso frente a la ganadería convencional, es la conservación de la fertilidad de los suelos donde se cría la especie. Desde el punto de vista ambiental, redunda el planteamiento de Sadeghian (2009):

La ganadería extensiva proporciona cambios negativos debido a la aridez del suelo, ya sea como producto de la deforestación en la creación de pastizales, al igual que por el pisoteo del ganado, que producen cambios estructurales, provocando detonantes erosivos, escases de nutrientes, entre otros, hasta la pérdida de diversidad de los mismos. 
Según Mora Marín et al., (2017), la problemática ambiental por la ganadería convencional extensiva en Colombia preocupa bastante, por cuanto los suelos se vienen afectando aceleradamente, donde el Estado debe intervenir con políticas y medidas que garanticen la conservación del recurso natural del suelo sin perder la productividad alimenticia.

La incertidumbre, producto de una actividad económica innovadora, pero sobre todo la falta de conocimiento y experiencia a nivel nacional sobre la helicicultura, son elementos que superan las dificultades y limitaciones que presentan los diferentes estudios realizados en el sector, siendo una oportunidad para proponer el modelo que logre generar una mayor eficiencia en la productividad de la carne de caracol, obteniendo al máximo los efectos que el mismo brindaría al ser humano y su entorno.

\section{DISCUSIÓN}

De acuerdo con las conclusiones de López-Palechor et al., (2017), no se puede traducir en la estagnación de la helicicultura en el país por las debilidades sujetas a la infraestructura logística y el conflicto armado, puesto que los entrevistados aluden poco conocimiento del tema, siendo un ejercicio más empírico que conlleva a desconocer otros modelos utilizados en países donde la misma actividad económica es sostenible.

Según Franco (2013), en la investigación realizada, se puede tener presente que si los inconvenientes para sacar adelante la actividad de la helicicultura son el conflicto armado y la infraestructura vial, es importante aclarar que Colombia se encuentra en proceso de posacuerdo, donde dicha actividad se convertirá en una alternativa sostenible para los colombianos, haciendo de esta manera factible el proyecto de la helicicultura en la región, sin dejar de lado los efectos o bondades que ella genera desde la innovación y desarrollo sostenible de una comunidad en general.

El costo unitario del kilo de carne de caracol actualmente oscila entre los 4.9 y los 3.6 dólares bajo el modelo de pronto engorde, logrando una optimización en el costo del producto del $26.53 \%$, siendo muy atractiva la helicicultura para los pobladores de una región.

Este costo unitario, sumado con el déficit de 56000 toneladas de la demanda pendiente por abastecer en un año de carne de caracol, según Martínez (2016), se convierte en la mejor oportunidad de motivación para crear más campos helicícolas en los municipios de Antioquia y, posteriormente, de Colombia.

Por último, el plano investigativo del mismo estudio determina que el impacto, desarrollo y beneficios para la economía y sociedad regional con las proyecciones realizadas son muy favorables, como lo señala Rodolfi (2016) y Forte et al., (2016). Además, el estudio sugiere que la actividad de la helicicultura no puede quedar en este punto, sino que se debe continuar la investigación en el tema para potencializar aún más la actividad helicícola que contribuya al sector primario de la economía.

Tal como lo refiere Vera Gacia (2016), las ventajas del desarrollo de la helicicultura en el sector primario de la economía, a partir de un modelo agropecuario sostenible, bajo el modelo de diamante expuesto por Porter, son una gran apuesta dentro del trabajo realizado por los autores, donde se 
evidencia una viabilidad integral de la actividad helicícola como oportunidad agroindustrial que fortalezca el crecimiento económico de la región.

\section{CONCLUSIONES}

Como alternativa social sostenible - inicialmente implementada en el municipio de San Vicente Ferrer -, la helicicultura puede ofrecer una oportunidad a los desmovilizados y a la población en condición de vulnerabilidad por el fenómeno del conflicto armado en Colombia. No obstante, para lograr lo anterior debe haber un conocimiento previo de la actividad helicícola y se debe contar con el apoyo del Estado colombiano, tomando como base la viabilidad integral de la actividad económica, la cual es un factor determinante desde cualquier punto de vista.

Partiendo precisamente de la viabilidad económica, el estudio de costeo y financiero de la helicicultura por el modelo convencional adoptado por varias regiones del municipio, hacen que la actividad económica sea poco atractiva, con un margen bruto por debajo del 20\%, lo que motiva a desistir en continuar con la misma. Esto conlleva a replantear el modelo actual por uno innovador, como es el caso de la estrategia «pronto engorde», que busca optimizar al máximo los recursos y/o factores de producción, reflejados, al mismo tiempo, en el nivel de producción, y estos a su vez en los estados financieros, generando un margen bruto proyectado que oscila entre el $37 \%$ y el $40 \%$.

Además, desde el campo medio ambiental, la helicicultura se convierte en una ganadería futurista que, a diferencia de la ganadería convencional, no afecta la fertilidad de los suelos y, adicionalmente, no generan gases de efecto invernadero que atenten a la población humana. El estudio realizado no solo pretende dar una solución desde lo económico como único atractivo, sino desde el contexto integral bajo el modelo de desarrollo sostenible.

Con respecto a lo social, el Estado colombiano debe generar una política pública donde participen todos los actores interesados en impulsar la producción de caracol como vía de ingresos a los desplazados y desmovilizados por el conflicto armado. Ya que, con la investigación realizada, se logró

evidenciar, como muestra inicial, la factibilidad que tiene el proyecto en el municipio de San Vicente Ferrer.

En general, a partir de la economía sostenible, y con el estudio realizado por la Universidad Nacional, debe destacarse la motivación y el compromiso mostrado por parte de los desmovilizados de las FARC, quienes en un $66.5 \%$ desean reintegrarse a la vida civil para desarrollar actividades pecuarias. Lo anterior conlleva a pensar que, en un futuro próximo, se puedan desarrollar investigaciones y proyectos alternativos en ganadería sostenible, pero no de mediano plazo, como la actual, que es la que principalmente afecta los recursos naturales, deteriorando su renovación y la conservación del medio ambiente.

\section{CONFLICTOS DE INTERÉS}

Los autores declaran que no presentan conflictos de interés financiero, profesional o personal que pueda influir de forma inapropiada en los resultados obtenidos o las interpretaciones propuestas. 


\section{CONTRIBUCIÓN DE AUTORES}

Para el desarrollo de este proyecto todos los autores han realizado una contribución significativa especificada a continuación:

Ricardo Alonso Colmenares-Flórez: conceptualización, diseño, desarrollo de la investigación, redacción y revisión final del manuscrito.

María Esther Alcántara-Gutierrez: conceptualización, revisión y ajustes de la versión final del manuscrito.

\section{REFERENCIAS}

Abay, K. A.; Jensen, N. D. (2020). Access to markets, weather risk, and livestock production decisions: Evidence from Ethiopia. Agricultural Economics, v. 51, n. 4, 577-593. https://doi.org/10.1111/agec.12573

ABC del Finkero. (2013, 10 de enero). Cría del caracol (Hélix Aspersa). URL

Álvarez-Gayou, J. (2005). Cómo hacer investigación cualitativa. Fundamentos y metodología. Paidós.

Arias, F. G. (1999). El proyecto de investigación. (3ra ed.). Editorial Episteme.

Artaraz, M. (2002). Teoría de las tres dimensiones de desarrollo sostenible. Ecosistemas, v. 11, n. 2. $\underline{U R L}$

Bojö, J.; Mäler, K.-G.; Unemo, L. (1990). Environment and Development: An Economic Approach. Springer Science \& Business Media.

Caballero, A. (2000). Metodología de la Investigación Científica: Diseño con hipótesis explicativas. Udegraf S.A.

Cairo, H.; Oslender, U.; Piazzini Suárez, C. E.; Ríos, J.; Koopman, S., Montoya Arango, V.; Rodríguez Muñoz, F. B.; Zambrano Quintero, L. (2018). "Territorial Peace": The Emergence of a Concept in Colombia's Peace Negotiations. Geopolitics, v. 23, n. 2, 464-488.

https://doi.org/10.1080/14650045.2018.1425110

Chacón Jiménez, P. C.; Maldonado Gutiérrez, A. A.; Toro Romero, A. A. (2010). Producción y comercialización de carne de caracol «HELIX ASPERSA MULLER» [Trabajo de grado, Universidad Minuto de Dios]. http://hdl.handle.net/10656/1745

Criollo-Uyaguari, A.; Feijóo-Valarezo, A.; Torres-Toukoumidis, A. (2020). La responsabilidad social corporativa de la TV local: estudio de caso. Revista CEA, v. 6, n. 12, 47-68. https://doi.org/10.22430/24223182.1583 
De Vincentiis, G. (2012). La evolución del concepto de desarrollo sostenible. Medio Ambiente \& Derecho: Revista electrónica de derecho ambiental, n. 23. URL

Drucker, P. F. (2004). La disciplina de la innovación. Harvard Bussines Review, v. 82, n. 8. URL

Erboz, G. (2020). A qualitative study on industry 4.0 competitiveness in Turkey using Porter diamond model. Journal of Industrial Engineering and Management, v. 13, n. 2, 266-282. https://doi.org/10.3926/jiem.2915

Eslava Gallo, O. L.; Mejía García, J. D.; García Ramírez, L.; Malagón Naranjo, J. C. (2008). Cría y comercialización de caracoles a través de la técnica de helicicultura [Trabajo de grado, Universidad de la Sabana]. $\underline{\text { URL }}$

Esterberg, K. G. (2002). Qualitative methods in social research. McGeaw-Hill.

Fantom, N.; Khokhar, T.; Purdie, E. (2016, 25 de abril). Ya se publicó la edición de 2016 de los Indicadores del Desarrollo Mundial: Tres características que no querrá perderse. URL

Fleta Zaragozano, J. (2017). El caracol como alimento y como terapia. Boletín de la Sociedad de Pediatría de Aragón, La Rioja y Soria, v. 47, n. 3, 67-72. URL

Forte, A.; Zucaro, A.; De Vico, G.; Fierro, A. (2016). Carbon footprint of heliciculture: A case study from an Italian experimental farm. Agricultural Systems, v. 142, 99-111.

https://doi.org/10.1016/j.agsy.2015.11.010

Franco Agudelo, S. (2013). Momento y contexto de la violencia en Colombia. Revista cubana de salud pública, v. 29, n. 1, 18-36. URL

Gallopín, G. (2003). Sostenibilidad y desarrollo sostenible: un enfoque sistémico. CEPAL. https://repositorio.cepal.org/handle/11362/5763

Gutiérrez, G. D.; Guzmán, A. C. (2009). La Helicicultura como posibilidad de creación de empresa en Colombia [Tesis de Especialización, Universidad de la Sabana]. https://intellectum.unisabana.edu.co/handle/10818/6460

Iglesias-de la Cruz, M.; Sanz-Rodríguez, F.; Zamarrón, A.; Reyes, E.; Carrasco, E.; González, S.; Juarranz, A. (2012). A secretion of the mollusc Cryptomphalus aspersa promotes proliferation, migration and survival of keratinocytes and dermal fibroblasts in vitro. International journal of cosmetic science, v. 34, n. 2, 183-189. URL

Jiménez Beltrán, S. M.; Rodríguez Castillo, S. L. (2007). Determinación de los procedimientos y procesos a seguir en la producción y comercialización del caracol helix aspersa para el mercado español, como alternativa agrícola y económica en Colombia [Trabajo de grado, Universidad de la Salle]. URL 
Kramer, M. R.; Porter, M. E. (2006). Estrategia y sociedad. Harvard Business Review, v. 84, n. 12, 4256. $\underline{U R L}$

Lamprea Delgado, Á.; Salazar Alonso, M.; Posada Arrubla, A. (2009). Formulación de la cadena productiva del caracol en Cundinamarca y Boyacá - Colombia para exportar hacia el mercado español. Revista U. D. C. A. Actualidad \& Divulgación Científica, v. 12, n. 1, 163-172. https://doi.org/10.31910/rudca.v12.n1.2009.652

López-Palechor, L. E.; Ruano-Ibarra, E. S.; Brisola, M. V. (2017). La helicicultura en Colombia: reforma normativa y rezago productivo. Apuntes del CENES, v. 36, n. 64, 225-250. https://doi.org/10.19053/01203053.v36.n64.2017.5420

Martínez, M. L. (2016, 21 de noviembre). Helicicultura. Agromeat. $\underline{\text { URL }}$

Meiselman, H. L. (2016). Quality of life, well-being and wellness: Measuring subjective health for foods and other products. Food Quality and Preference, v. 54, 101-109. https://doi.org/10.1016/i.foodqual.2016.05.009

Mora Marín, M. A.; Ríos Pescador, L.; Ríos Ramos, L.; Almario Charry, J. L. (2017). Impacto de la actividad ganadera sobre el suelo en Colombia. Ingeniería y Región, v. 17, 1-12. https://doi.org/10.25054/22161325.1212

Organización de las Naciones Unidas para la Alimentación y la Agricultura. (2016). El estado mundial de la agricultura y la alimentación. Cambio climático, agricultura y seguridad alimentaria. FAO. $\underline{U R L}$

Padilla, E. (2002). Equidad intergeneracional y sostenibilidad: las generaciones futuras en la evaluación de políticas y proyectos [Tesis de doctorado, Universitat Autònoma de Barcelona]. https://hdl.handle.net/10803/3986

Red Andaluza de Experimentación Agraria. (2008). Ganadería: Helicicultura. Campaña 2008. Instituto de Investigación y Formación Agraria y Pesquera. $\underline{\text { URL }}$

Rodolfi, P. E. (2006). Selección de mercados europeos para la exportación de caracoles [Tesis de doctorado, Universidad Empresarial Siglo 21].

https://repositorio.uesiglo21.edu.ar/handle/ues21/12180

Sadeghian, K. (2009). Impacto de la ganadería sobre el suelo. Obtenido de alternativas sostenibles de manejo. $\underline{U R L}$

Sastre, R. (2006). Helicicultura. Cría de caracoles en la Argentina: una alternativa innovadora en agronegocios [Tesis de Maestría, Universidad de Buenos Aires]. URL

Valles, M. S. (2000). Técnicas cualitativas de investigación social. Reflexión metodológica y práctica profesional. Síntesis Editorial. 
Vera Garcia, R. E. (2016). Microbiología del caracol Helix Aspersa Müller. Aplicaciones biotecnológicas para su mejoramiento sanitario con impacto en su comercialización [Tesis de doctorado, Universitat Autònoma de Barcelona]. URL

Villamil Kiewu, M. (2010). Los caracoles, una oportunidad de negocio. Dinero. URL 